Article

\title{
Unidirectional Optical Kerr Transmittance in Hierarchical Carbon/Platinum Nanostructures
}

\author{
Samuel Morales-Bonilla ${ }^{1,2}$, Cecilia Mercado-Zúñiga ${ }^{3}$ (D), Juan Pablo Campos-López ${ }^{2}$, \\ César Carrillo-Delgado ${ }^{4}$, Claudia Lizbeth Martínez-González ${ }^{5}$ and Carlos Torres-Torres ${ }^{5, * \text { (D) }}$ \\ 1 Ingeniería en Sistemas Automotrices, Escuela Superior de Ingeniería Mecánica y Eléctrica Unidad \\ Culhuacán, Instituto Politécnico Nacional, Ciudad de México 04440, Mexico; smoralesbo@ipn.mx \\ 2 División de Ingeniería en Mecatrónica, Universidad Politécnica del Valle de México, Tultitlán, \\ Estado de México 54910, Mexico; campos.dono@gmail.com \\ 3 Depto. Ing. Materiales, Tecnológico de Estudios Superiores de Coacalco, Coacalco de Berriozábal, \\ Estado de México 55700, Mexico; cecilia@tesco.edu.mx \\ 4 Depto. de Ingeniería Robótica, Universidad Politécnica del Bicentenario, Silao, Guanajuato 36283, Mexico; \\ ccarrillop@upbicentenario.edu.mx \\ 5 Sección de Estudios de Posgrado e Investigación, Escuela Superior de Ingeniería Mecánica y Eléctrica \\ Unidad Zacatenco, Instituto Politécnico Nacional, Ciudad de México 07738, Mexico; clmartinezg@ipn.mx \\ * Correspondence: ctorrest@ipn.mx or crstorres@yahoo.com.mx
}

Received: 2 July 2020; Accepted: 28 July 2020; Published: 30 July 2020

check for updates

\begin{abstract}
A strong contrast in the third-order nonlinear optical effects exhibited by hierarchical nanostructures explored in a bidirectional optical circuit is reported. The samples were integrated by multiwall carbon nanotubes and platinum-decorated carbon nanotubes synthetized by an aerosol pyrolysis technique and followed by a chemical vapor deposition method. Coupled and decoupled third-order nonlinear optical properties of the nanocomposites were studied. A nanosecond two-wave mixing experiment at $532 \mathrm{~nm}$ wavelength was conducted to analyze the optical Kerr effect in the samples. Multi-photonic interactions were evaluated by a single-beam transmittance as a function of input irradiance and volume fraction of the nanoparticles integrated in the nanohybrids. A two-photon absorption process was identified as the main physical mechanism responsible for the anisotropy in the observed optical nonlinearities. Random carbon nanotube networks in film form were put on top of platinum-decorated carbon nanotubes in order to build up a bilayer sample featuring optical selectivity. The switching of optical signals in propagation through the samples was obtained by an orientation-selectable optical transmittance. Unidirectional optically controlled laser pulses dependent on irradiance and polarization in a two-wave mixing was proposed with potential nanophotonic and nanoelectronic applications. The design of signal processing functions driven by nanohybrid platforms can be contemplated.
\end{abstract}

Keywords: nonlinear optics; two-wave mixing; sensors; carbon nanotubes; platinum nanoparticles

\section{Introduction}

In the last years, the progress in nanotechnology has promoted outstanding solutions for developing new alternatives in material science and engineering. A growing number of different nanoparticles have been demonstrated to be useful in multifunctional applications regarding their remarkable nonlinear optical and mechanical characteristics [1-7].

In this aspect, the advanced features of carbon-based nanomaterials pointed out unique geometrical structures, high-speed electronic phenomena, photonic nonlinearities, and superlative mechanical effects [8-11]. Nanostructures can behave differently from macrostructures due to the presence of significant size effects that are not present at the macro scale, i.e., surface stresses, strain gradients, 
and non-locality [12]. Particularly, carbon nanotubes (CNT) take advantage of their size and shape to present an exceptional mechanical response under the influence of external agents and resonance effects that may be useful to study pretty small deformations at the order of nanometers [13].

A substantial effort has been made in order to analyze the structural nature composed by carbon atoms linked in hexagonal shapes and containing several hexagonal shapes conformed in concentric nanotubes [14]. When the length of CNT is several times larger than their inner diameter, they behave as electrical dipoles for a specific wavelength, and then, a strong selectivity for optical absorption can be originated [15]. In this direction, multiwall CNT (MWCNT) can absorb approximately 3 times the amount of light absorbed by single-wall CNT (SWCNT) because of the presence of more electrons available for absorption per particle [16]. Furthermore, the electrical and optical behavior exhibited by CNT can be enhanced by the modification in their chirality and alignment [17].

Moreover, hierarchical carbon nanostructures have been attractive as a platform for the fabrication of plasmonic-based sensors. The light-to-physical conversion efficiencies of CNT have been tailored to be significantly larger for specific quantum processes derived from metal decoration methods [18]. In addition, polarization and irradiance conditions can be chosen to produce multivalent effects that can be explained by simple fractional models in real applications [19].

By using chemical deposition methods, a good control on the parameters of synthesis in noble metal-decorated carbon nanostructures has been obtained [20]. Exhaustive studies have been carried out in the nonlinear optical properties of noble metal nanoparticles integrated in nanocomposites [21]. Nevertheless, the optical properties in platinum nanoparticles (Pt NPs) are still weakly reported [22]. Even though the peak in the absorption band of the Surface Plasmon Resonance (SPR) of platinum is at $215 \mathrm{~nm}$ [23], it is still observed to have saturable absorption at lower fluences, which are usually linked to SPR in gold nanoparticles (532 nm) [24]. Fuel cells, electronic signal modulation, photonic sensing, and nanomechanical actuators are some of the other fascinating applications that can be contemplated using carbon-based nanohybrids [25]. The nonlinear optical response exhibited by carbon/metal nanostructures can play a key role in a wide number of scientific disciplines related to photoelectrical devices [26] and all-optical instrumentation systems [27], among others.

Therefore, considering the arguments mentioned above, this research has been devoted to further investigate the vectorial effects produced by nonlinear optical phenomena in CNT. The influence of platinum decoration in the studied CNT allowed us to present hierarchical nanostructures with orientation-selectable nonlinear optical response. A modification in the optical and mechanical effects exhibited by CNT samples was numerically and experimentally analyzed. The nanosecond third-order nonlinear optical behavior of the samples was evaluated by considering discrete groups of energy automatically modulated by off-resonance pulses in two-wave mixing experiments. The potential use of carbon-based nanostructures promises the development of good candidates for designing sensors and instrumentation nanodevices [28,29]. The nonlinear optical effects exhibited by MWCTs have been suggested for optical instrumentation [30] and signal processing [31]. Remarkably, the tuning of refractive and absorptive effects by intense light can be useful for flexible optical platforms [32]. Moreover, optical nonlinearities can be contemplated for implementing all-optical systems sensitive to the vectorial nature in the superposition of optical waves [33]. The significance of this study pointed out third-order nonlinear optics in carbon/metal nanohybrids for interferometrically controlled functions [34,35].

\section{Materials and Methods}

\subsection{Sample Preparation}

For the preparation of the CNT, an aerosol pyrolysis method was employed [35]. The aerosol was ultrasonically generated, and an argon flow of $2.5 \mathrm{l} / \mathrm{min}$ was employed. Toluene and ferrocene in a carbon solution were used in the synthesis. The resulting solution in saturation condition was 
deposited in quartz tube at $800{ }^{\circ} \mathrm{C}$ in order to produce a stable transport in the precipitation effect and to guarantee the growing of the nanotubes.

In order to include platinum nanoparticles in the CNT samples, a chemical vapor deposition technique was used. The CNT samples located in a horizontal quartz tube reactor at about 5-7 Torr received two sequential thermal processes. As a part of the process, a mixture of $\mathrm{Pt}$ precursor [ $\left(\mathrm{CH}_{3}-\mathrm{COCHCO}-\mathrm{CH}_{3}\right)_{2} \mathrm{Pt}$; Aldrich $97 \%$ ] was incorporated by grinding with agate mortar and pestle for $10 \mathrm{~min}$. The resulting mixture under Ar gas flow $\left(100 \mathrm{~cm}^{3} / \mathrm{min}\right)$ was heated at $180^{\circ} \mathrm{C}$ for $10 \mathrm{~min}$ and then at $400{ }^{\circ} \mathrm{C}$ for $10 \mathrm{~min}$. The morphology and structure of the samples was analyzed by Transmission Electron Microscopy (TEM; JEM 220FS) and Scanning Electronic Microscopy (SEM; SEM ULTRA 55 FEG System from ZEISS with Secondary Electron and Backscattering Detector). Compositional analysis was carried out by Energy-Dispersive X-ray Spectroscopy (EDS; JEOL JSM-6701F). The UV-vis spectra of the samples suspended in ethanol was recorded by a USB 2000+XR1-ES spectrometer assisted by a DH-2000 light source with a 300-900 $\mathrm{nm}$ wavelength of emission. The nonlinear optical experiments were performed with samples in film form with approximately $50 \mu \mathrm{m}$ thickness and deposited on quartz substrates. The MWCNT film was put on top of the Pt decorated MWCNT (Pt-MWCNT) in order to integrate a bilayer sample for the nonlinear optical measurements.

\subsection{Nanosecond Transmittance and Bidirectional Two-Wave Mixing Experiments}

A Neodymium-Yttrium Garnet (Nd-YAG) laser system Continuum Model SL II-10 emitting pulses of 4 nanosecond in single-shot mode at $532 \mathrm{~nm}$ wavelength was used as an optical source to study the nonlinear optical response of the samples. Single-beam experiments were carried out in order to observe any nonlinear optical absorption exhibited by the samples. A vectorial two-wave mixing method [36] was used to analyze the nonlinear refraction and nonlinear absorption in the samples irradiated by the nanosecond pulses. A schematic illustration associated with the implementation of the two-wave mixing can be observed in Figure 1. L1-3 represent the lenses that focused the spot size in the sample to be approximately $0.1 \mathrm{~mm}$. The beam splitters BS1-2 separate the principal beam into the pump beam and two probe beams with linear polarization. The beams were reflected by mirrors M1-9 to follow different optical paths. A half-wave plate, $\lambda / 2$, was used to rotate the polarization of the pump beam during the experiment, while the polarization of both probe beams was fixed. The modulation of the polarization of each probe beam by the participation of the pump beam was separately analyzed by the polarizers, A1-3, with its transmission axis in the orthogonal position with respect to the initial polarization of the probe beam. It means that the probe beam traveling from right to left (forward direction) was blocked during the measurement of the probe beam traveling from left to right (backward direction), and vice versa. The transmitted irradiance was measured by photodetectors PD1-3. The two-wave mixing experiments were carried out in both backward and forward directions in respect to the incidence in the bilayer sample with the MWCNT in the right part of the experimental setup and the Pt-MWCNT in the left part. This was made in order to see if a change in the transmittance of the probe beams depends on the zone of the sample that interacts first with the beam, MWCNT, or Pt-MWCNT. We noticed an increase in the probe transmittance in the two-wave mixing experiment by using geometrical angles between interacting beams close to $1^{\circ}$, as it has been previously reported for Kerr media [37]. However, an increase in the error bar was also promoted as a result of an increase in the scattering emerging from our sample in the Raman-Nath diffraction regime. With these considerations, the geometrical angle between beams was systematically settled at $10^{\circ}$ according to our evaluation of the relation of signal-to-noise rate in the experimental setup. The stability of the pulse at the output of our laser system employed in the experiments was about $\pm 3 \%$. Regarding that the transmittance in the two-wave mixing is a third-order nonlinear optical process, there is an error bar that is larger as a consequence of the nonlinearity involved in the studied optical processes. The error bar in our transmittance experiments and two-wave mixing is approximately $\pm 10 \%$. Each point in the experimental data related to nonlinear transmittance experiments and two-wave mixing experiments corresponds to an average of 10 shots. 


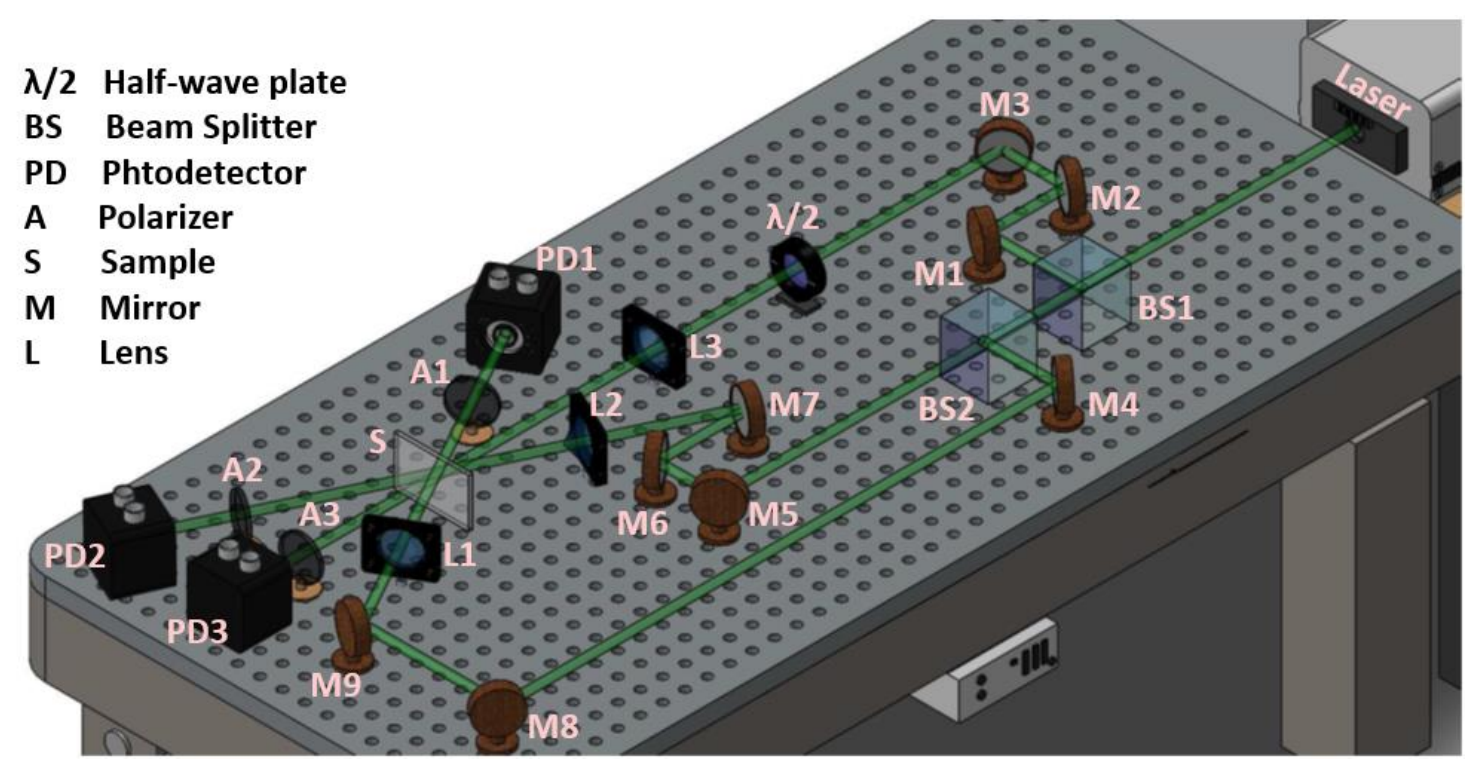

Figure 1. Schematic illustration of the two-wave mixing experiment.

The numerical estimation of the transmitted irradiances was approximated by using the wave equation [38]:

$$
\nabla^{2} E_{ \pm}=-\frac{n_{ \pm}^{2} \omega^{2}}{c^{2}} E_{ \pm}
$$

where the electric fields in propagation through the samples are represented by the circular components of the right and left electric fields $E+$ and $E-$, respectively. The optical frequency of the light is $\omega$, the index of refraction is $n$, and the speed of the light is $c$. We consider a refractive index dependent on irradiance that can be approximated as follows [38]:

$$
n_{ \pm}^{2}=n_{0}^{2}+4 \pi\left(\chi_{1122}^{(3)}\left|E_{ \pm}\right|^{2}+\left(\chi_{1122}^{(3)}+\chi_{1212}^{(3)}\right)\left|E_{\mp}\right|^{2}\right)
$$

the weak-field refractive index is represented in Equation (2) as $n_{0}$, and the independent components of the third-order optical susceptibility tensor $\chi^{(3)}$ are $\chi_{1122}^{(3)}$ and $\chi_{1212}^{(3)}$. The calibration of the two-wave mixing experiment was conducted by using a $\mathrm{CS}_{2}$ sample with the magnitude of its third-order nonlinear optical susceptibility, $\left|\chi^{(3)}\right|=1.9 \times 10^{-12}$ esu [38], contained in a quartz cuvette with 1 mm length.

The real and imaginary parts of the complex magnitude associated with $\chi^{(3)}$ can be given by [38],

$$
\chi_{1111}^{(3)}=\frac{n_{0} c}{7.91 \times 10^{2}} n_{2}+i \frac{n_{0}^{2} c \lambda}{\pi^{2}} \beta
$$

where $\lambda$ is the optical wavelength, $n_{2}$ is the nonlinear refractive index, and $\beta$ the nonlinear optical absorption coefficient.

The mathematical description for the transmitted irradiance, $I$, as a function of the propagation distance $L$, with incident irradiance $I_{0}$ through a nonlinear optical absorptive media is:

$$
I(L)=\frac{I_{o} \exp \left(-\alpha_{o} L\right)}{1+\beta I_{o} L_{e f f}},
$$

with $\alpha_{0}$ as the optical absorption coefficient al low irradiance, and $L_{\text {eff }}$ as the effective length given by the mathematical expression:

$$
L_{e f f}=\frac{\left(1-\exp \left(-\alpha_{0} L\right)\right)}{\alpha_{0}}
$$


In order to analyze the contribution of different elements integrated in a hybrid nanostructure, the magnitude of $\chi^{(3)}$ can be approximated taking into account the relation in volume fraction, $\rho$, in the nanostructures as follows,

$$
\chi_{m+s}^{(3)}=(1-\rho) \chi_{m}^{(3)}+\rho \chi_{s}^{(3)}
$$

where $\chi_{m+s}^{(3)}$ represents the nonlinear third-order susceptibility of the integrated nanohybrids with $\chi_{m}^{(3)}$ and $\chi_{s}^{(3)}$ as the correspondent values of the uncoupled nanostructures.

\section{Results and Discussion}

Figure 2a depicts a typical TEM image of the CNT samples in bright field mode confirming the multiwall nature of the nanotubes. The darker array in the micrograph represents the inner diameter (d) of a representative CNT, and the surrounding layers represent multiwall layers that form the outer diameter (D). The distance between the inner and outer diameter is the thickness $(t)$ of the MWCNT. Figure $2 b$ shows an isolated pristine CNT in a SEM image. The length $L$ of a tube can be seen. Figure $2 c$ shows an SEM micrograph of a representative Pt-decorated MWCNT sample; the bright points in the image correspond to the Pt nanoparticles incorporated in the walls of the tubes. Figure $2 \mathrm{~d}$ shows the EDS analysis revealing the presence of Pt in the metal-decorated CNT samples and confirming the concentration of the metal in the nanostructures.

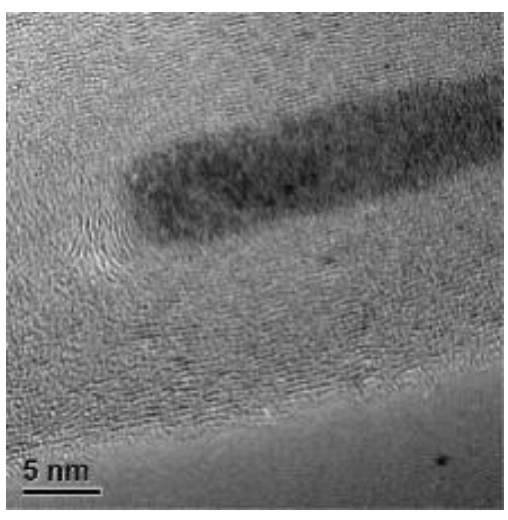

(a)

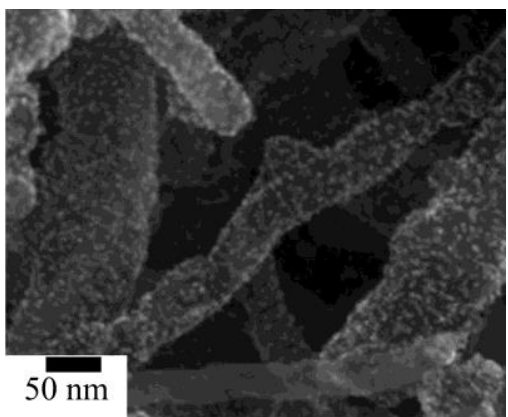

(c)

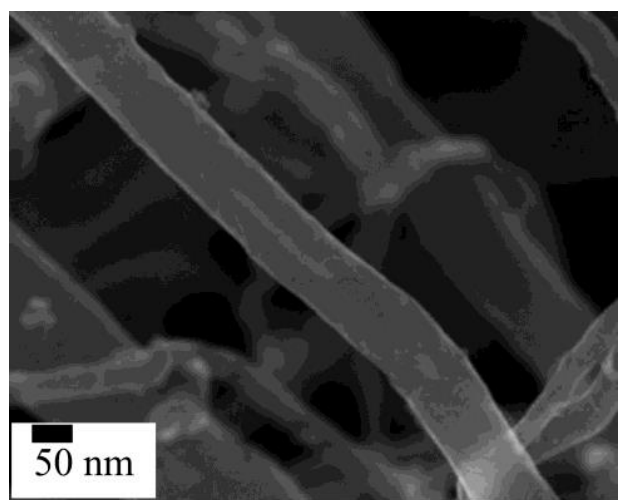

(b)

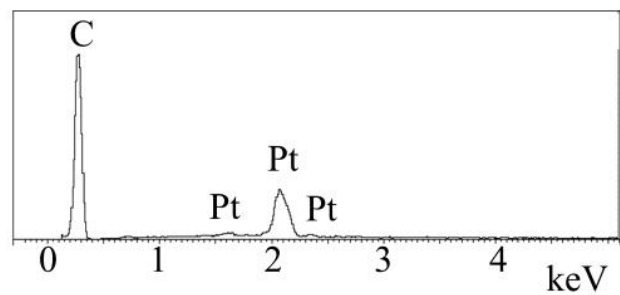

(d)

Figure 2. (a) TEM image of a typical carbon nanotubes (CNT) concerning the studied sample; (b) SEM image of an isolated pristine CNT; (c) SEM image of platinum-decorated multiwall CNT (Pt-MWCNT); (d) Energy-Dispersive X-ray Spectroscopy (EDS) in a representative section of Pt nanoparticles supported on a CNT bundle.

Similar optical absorption spectra were acquired during the evaluation of the MWCNT and Pt-MWCNT samples in a liquid suspension; both spectra are comparatively equal. A concentration of 1 
$\mathrm{mg}$ of nanostructures in $5 \mathrm{~mL}$ of ethanol was selected in order to clearly see the peak in the absorption band of the samples. Figure 3 plots the typical UV-vis absorption spectrum of the studied Pt-MWCNT samples where it is possible to see close to the $270 \mathrm{~nm}$ wavelength the resonant response associated with the $\pi-\pi$ bond of the carbon nanostructures. This UV region also corresponds to the wavelengths where the absorption peak of the Localized Surface Plasmon Resonance exhibited by Pt nanoparticles could emerge [39].

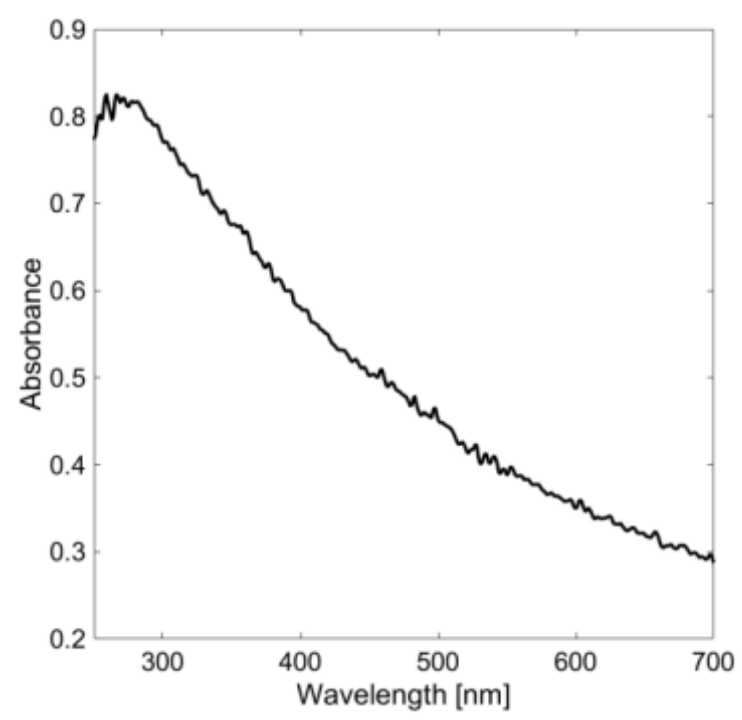

Figure 3. Typical UV-vis absorbance spectrum of the studied samples.

An ablation threshold of $110 \mathrm{~mJ} / \mathrm{cm}^{2}$ in the samples was experimentally measured at a $532 \mathrm{~nm}$ wavelength with $4 \mathrm{~ns}$ pulse duration in single-shot mode. We verified that thermal damage can be derived by heat propagation up $50{ }^{\circ} \mathrm{C}$ in the samples conducted by a Thermo-Scientific CIMAREC system (model SP131635), which was assisted by an infrared pyrometer (Master Instruments model MI-1326S). However, no important changes in temperature were detected in the samples irradiated by off-resonance optical pulses in single-shot mode at $532 \mathrm{~nm}$ wavelength below $20 \mathrm{MW} / \mathrm{cm}^{2}$. Then, we analyzed if a mechanical action induced by high-irradiance pulses could be promoted in the nanotubes. It is known that the physical properties of CNT are sensitive due to their diameter, length, and chirality. These values have a strong influence on the electronic properties of CNT. It has been shown that CNT can be either metallic or semi-conducting materials [40]. With this in mind, we assumed that the geometry of CNT can be defined by the internal tube diameter (d), the external tube diameter (D), a longitudinal length (L), a total thickness ( $t$ ), and a circular cross-section. The ratio $\mathrm{L} / \mathrm{D}$ is called slenderness ratio of the tube. The lowest length $\mathrm{L}$ of CNT was close to $180 \mathrm{~nm}$ according to our statistical TEM observations. For simplicity, we used a 12 carbon atoms configuration at each layer in our numerical simulations. It is worth mentioning that the mechanical behavior of CNT under the influence of external factors has been described by many techniques; but essentially, these techniques correspond to ab initio processes, quantum mechanics, and Finite Element Method (FEM) calculations by using discrete and continuous models [41,42]. In this paper, in order to describe the specific geometry properties, we took into account the main considerations described by Fan, et al. [43]. We used the discrete FEM method taking into account each element as a mechanical beam in order to form a zig-zag carbon layer. We considered 1129 beam elements type B33 to determine the final geometry of the internal cylinder [44]. The software used in the simulations was ABAQUS. L/D was the principal geometrical parameter to evaluate the deformation of the tube. Thus, carbon atoms were considered as nodes, and the distances between every two atoms were treated as mechanical beams with an initial distance equivalent to the covalent bond. Thus, the carbon-carbon bond length $\left(\alpha_{c-c}\right)$ was assumed to be $0.142 \mathrm{~nm}$ [45]. However, no established values are available for the wall thickness of 
an isolated nanotube. The values of thickness suggested by available literature varied significantly from $0.066 \mathrm{~nm}$ [46] to $0.68 \mathrm{~nm}$ [47]. In view of such a wide range of suggested thickness, our study was complemented using TEM observations to deduce this value. Our observations indicate that a MWCNT had about 44 walls with a total thickness of $15 \mathrm{~nm}$ (see Figure 2a), thus, an appropriate wall thickness from a CNT. Taking into account our TEM observations, we conclude that the value of e must be near $0.341 \mathrm{~nm}$. This value is in agreement with the thickness $0.335 \mathrm{~nm}$ reported in the literature [48]. The effect of Van der Waals interaction over the vibration characteristics of MWCNT is crucial for describing physical effects emerging from carbon nanostructures [49]. Particularly, the fundamental mechanical properties exhibited by MWCNT can be analyzed by considering linear vibrations exhibited by concentric SWCNT with Van der Waals interactions between each pair of layers [50]. Concerning the cross circular section of MWCNT, we observed a range of diameters from 1 to $10 \mathrm{~nm}$; thus, we considered an average diameter of $5 \mathrm{~nm}$ and a length $\mathrm{L} \geq 180 \mathrm{~nm}$. A unit scaling processor was used to reduce the numerical error due to the very small dimensions of the results. By doing this, we obtained similar results from Li, et al. [42].

We also consider that our sample in film form could present a strain in the CNT when it is irradiated by a fluence close to the photodamage. The action of light over whole cylinders shut on the global system, thus, an increment in sensitivity due to excitations of electrons can be expected per unit of length. In any case, when the global system is modulated by a mechanical effect, changes in electromagnetic parameters must be also present. Samples integrated by CNT strongly absorb the energy associated with vibration of electrons as a result of laser irradiance taking into account the multilayer configuration; the energy transferred to each carbon layer modifies their geometric configuration due to a strain effect. Besides, inhomogeneous electromagnetic irradiance is able to induce an additional internal motion. Remarkably, the range of these values depends on the optical irradiance before reaching optical ablation. In this direction, we also evaluated the critical load, $P_{\mathrm{cr}}$. In this sense, our concern will be with the amount of energy coming from the laser system as long as $P$ does not exceed the critical value $P_{\text {cr }}$. Figure 4 a describes the values of $P$ and also $P_{\text {cr }}$ versus $\mathrm{L} / \mathrm{D}$ that are related to permanent mechanical deformation and optical ablation conditions. Within this figure, we also sketch two scenarios, (1) a quasilinear behavior (green line) that delimits a plastic deformation of two cylinders before reaching melting and plastic deformation conditions, and (2) a plastic deformation in which ablation and $P>P_{\text {cr }}$ could be expected. Figure $4 \mathrm{~b}$ delimits the geometry of an isolated carbon cylinder with a particular thickness. Figure $4 \mathrm{c}$ illustrates the cross circular section of MWCNT. We note from Figure 4 that $P$ is inversely proportional to L/D. For a typical mechanical behavior in the sample, we can assume that critical stress $\left(\sigma_{c r}\right)$ will occur across thickness.

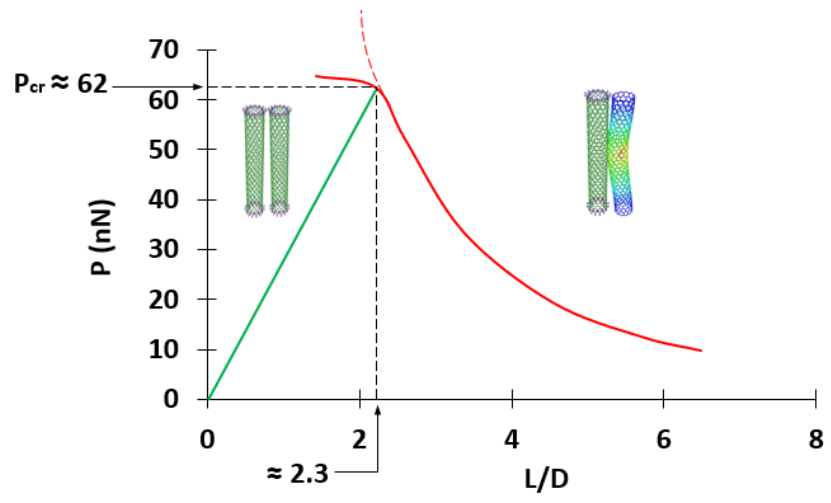

(a)

Figure 4. Cont. 


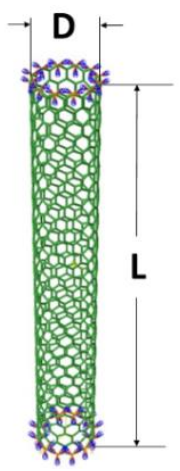

(b)

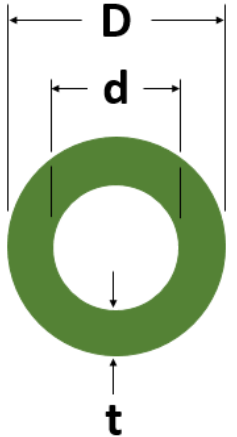

(c)

Figure 4. (a) Plot of slenderness ratio vs. load; (b) schematic representation of an isolated nanotube; (c) outer diameter D and inner diameter d of MWCNT.

It should be noted that the condition $\mathrm{L} / \mathrm{D} \geq 2.3$ can be related to the potential buckling in the structure of the tube due to the action of nanosecond pulses with irradiances below the ablation threshold of the samples. In this case, our concept of buckling refers to the excitation of electrons from the inner diameter to the external diameter of MWCNT to produce permanent deformations and photodamage. It is worth mentioning that these opto-mechanical idealistic results obey the Euler's critical load for buckling defined by [51].

$$
\frac{P_{c r}}{A}=\frac{\pi^{2} E}{(L / r)^{2}}
$$

In this case, the effect of the value $P_{\mathrm{cr}} / A$ becomes negligible for large values of $\mathrm{L} / \mathrm{D}$. Furthermore, we should keep in mind that the right position of loading $P$ as a result of light propagation is seldom known with a high degree of precision. However, in this idealistic approach, we initially evaluated the value of the load, $P$, for $\mathrm{CNT}$ in a rigid condition. It can be considered that increments in temperature generate a reduction in the magnitude of $P$. Furthermore, if $P$ is not aligned (the most probably scenario), the mechanical momentum might increase the sensitivity of the CNT.

From Figure $4 \mathrm{a}$, we also observe that values $P \leq 62 \mathrm{nN}$ and $\mathrm{L} / \mathrm{D} \leq 2.3$ describe a quasi-linear mechanical behavior exhibited by the sample studied. On the contrast, if $\mathrm{L} / \mathrm{D}>2.3$, it gives origin to a deformation and a possible melting process. This result can be related to interatomic forces between carbon atoms in cylinder layers and a direct consequence of the geometrical parameters associated with $\mathrm{D}$ and L. A proportional relation between Young's modulus and the values of diameters $\mathrm{D}-\mathrm{d}$ can be expected in the sample. A high Young's modulus will be present for a low nanotube internal diameter $\mathrm{d}$; this is attributed to an increment in cross-sectional area $(A)$ to transmit the load as a consequence of an increment of thickness $t$ from MWCNT.

From our TEM explorations concerning MWCNT, we estimated the following geometrical values: $\mathrm{D}=134 \mathrm{~nm}, \mathrm{~d}=4 \mathrm{~nm}, \mathrm{~L}>180 \mathrm{~nm}$, and thickness $15 \mathrm{~nm}$. The Young's modulus from these results is in a range from $0.81 \mathrm{TPa}$ to $1.28 \mathrm{TPa}$; thus, we assumed that our predictions are within reasonable agreement with previous results in carbon nanostructures [52]. On the other hand, our results from the computational technique are also comparable to those provided by different models reported with magnitudes from 1.0 to 1.24 TPa [53]. One advantage of this analysis is the simplicity of the working principle based on the classical structural mechanics and experimental observations.

Standard two-wave mixing measurements were conducted by using the setup illustrated in Figure 1 in order to evaluate the magnitude of $\chi^{(3)}$ in the nanostructures integrated in film form. Figure 5 shows the experimental results as a function of the variation of the volume fraction of nanoparticles of $\mathrm{Pt}$ decorating MWCNT in the sample studied in film form. The numerical fitting was made by using Equation (1). The estimated value for the MWCNT was $\chi_{m}^{(3)}=2.2 \times 10^{-9} \mathrm{esu}$, and for 
the Pt nanoparticles, $\chi_{s}^{(3)}=2.76 \times 10^{-8}$ esu according to a decoupling approximation for the nonlinear optical response of composite media expressed by Equation (6).

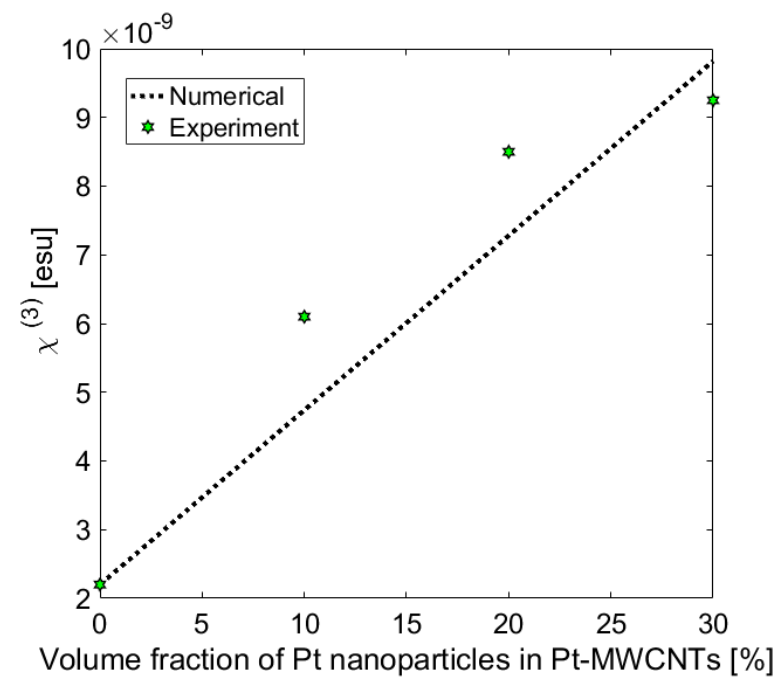

Figure 5. Magnitude of $\chi^{(3)}$ as a function of a change in the volume fraction of nanoparticles of $\mathrm{Pt}$ decorating MWCNT in a sample in film form.

From Figure 5, it can be clearly observed that the incorporation of 30\% of the volume fraction of $\mathrm{Pt}$ nanoparticles in the MWCNT can improve the nonlinearity by a factor of almost four. However, it is remarkable that the estimated optical nonlinearity $\chi_{s}^{(3)}$ of the metal nanoparticles seems to be one order of magnitude higher than the same parameter exhibited by the MWCNT denoted as $\chi_{m}^{(3)}$.

In order to resolve the imaginary part related to $\chi^{(3)}$, we explored an input-output experiment as a function of optical irradiance and volume fraction of the Pt nanoparticles incorporated in the MWCNT samples. The experimental results are depicted in Figure 6. The numerical fitting of $\beta$ obtained by Equation (4) is shown in Table 1 together to the $n_{2}$ coefficients estimated by Equation (3) and the results of $\chi^{(3)}$ measured in this research.

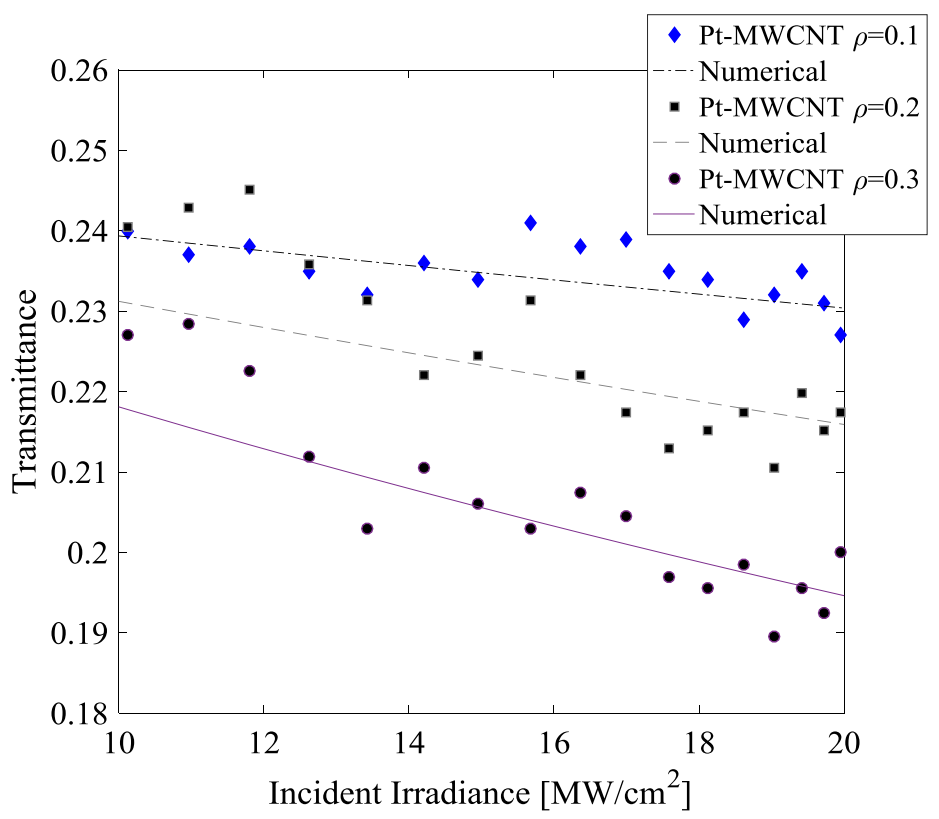

Figure 6. Magnitude of $\chi^{(3)}$ as a function of a change in the volume fraction of nanoparticles of Pt decorating MWCNT in a sample in film form. 
Table 1. Nonlinear optical parameters evaluated in nanohybrids studied at a $532 \mathrm{~nm}$ and $4 \mathrm{~ns}$ pulses.

\begin{tabular}{cccc}
\hline $\begin{array}{c}\rho \text { Related to Pt Nanoparticles } \\
\text { Incorporated to }\left[\mathbf{m}^{2} / \mathbf{W}\right]\end{array}$ & $\begin{array}{c}\boldsymbol{n}_{\mathbf{2}} \\
{\left[\mathbf{m}^{\mathbf{2}} / \mathbf{W}\right]}\end{array}$ & $\begin{array}{c}\boldsymbol{\beta} \\
{[\mathbf{m} / \mathbf{W}]}\end{array}$ & $\boldsymbol{\chi}^{(3)}[\mathbf{e s u}]$ \\
\hline 0 & $-1.46 \times 10^{-15}$ & - & $2.21 \times 10^{-9}$ \\
0.1 & $-4.03 \times 10^{-15}$ & $3.1 \times 10^{-9}$ & $6.10 \times 10^{-9}$ \\
0.2 & $-5.62 \times 10^{-15}$ & $5.9 \times 10^{-9}$ & $8.53 \times 10^{-9}$ \\
0.3 & $-6.08 \times 10^{-15}$ & $10.9 \times 10^{-9}$ & $9.25 \times 10^{-9}$ \\
\hline
\end{tabular}

It is interesting that the strong $n_{2}$ exhibited by the pure MWCNT can be considered for envisioning all-optical modulation applications; however, the incorporation of the metal nanoparticles causes an increase in $n_{2}$, but also, a detriment derived by $\beta$ automatically emerges. With this implication, we designed a bilayer sample with Pt-MWCNT and pure MWCNT in order to obtain the advantage of a dissimilar bidirectional response for switching functions.

Vectorial two-wave mixing experiments were carried out by using the setup illustrated in Figure 1 in order to identify the modification of the probe transmittance by the pump beam action. The experimental setup was calibrated by using a standard nonlinear $\mathrm{CS}_{2}$ sample with a nonlinear refractive index $n_{2}=6 \times 10^{-14} \mathrm{~cm}^{2} / \mathrm{W}$ [38]. Figure 7a shows the evolution of a single-beam transmitted irradiance as a function of its incident irradiance; while Figure $7 \mathrm{~b}$ plots the transmitted probe irradiance as a function of the angle between the planes of linear polarization of the incident beams. The best fitting of the experimental data were calculated by Equation (1) and the beam propagation method. Statistical measurements were conducted in order to estimate the third-order nonlinear optical parameters shown in Table 2 for the bilayer film in the forward and backward direction of irradiation for the bilayer film with an error bar of about $\pm 10 \%$. These results are in good agreement with previous publications $[30,54]$.

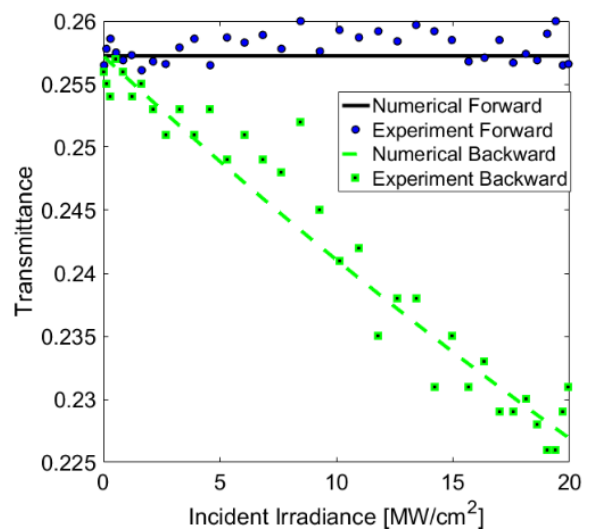

(a)

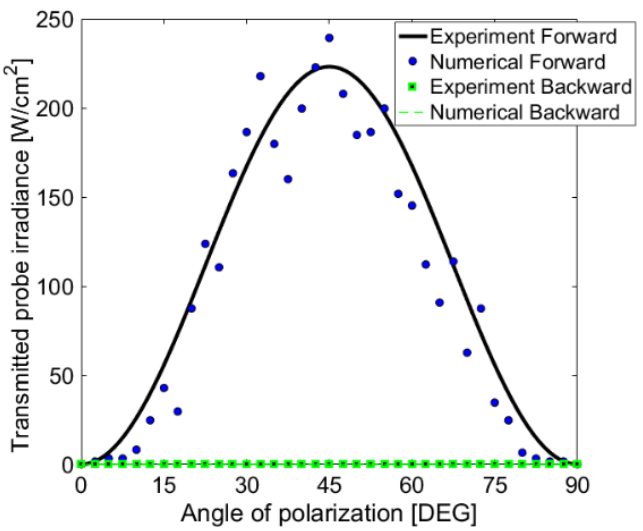

(b)

Figure 7. (a) Single-beam transmittance as a function of the incident irradiance; (b) transmitted probe irradiance as a function of the angle between planes of polarization of the incident beams.

Table 2. Nonlinear optical parameters evaluated in the samples studied at $532 \mathrm{~nm}$ and $4 \mathrm{~ns}$ pulses.

\begin{tabular}{ccc}
\hline Sample Incidence & $\boldsymbol{n}_{\mathbf{2}}\left(\mathrm{cm}^{2} / \mathrm{W}\right)$ & $\boldsymbol{\beta}(\mathrm{cm} / \mathrm{W})$ \\
\hline Forward & $-1.12 \times 10^{-11}$ & - \\
MWCNT/Pt-MWCNT & & $4.9 \times 10^{-7}$ \\
Backward & - & \\
Pt-MWCNT/MWCNT & &
\end{tabular}

The data shown in Figure 7a represent a clear signature of a two-photon absorption process in the backward direction of the experiment as a decrease in the transmittance as a function on irradiance [38], 
while the inhibition of the nonlinear optical absorption seems to occur if the sample is explored in the forward direction. Conversely, Figure $7 \mathrm{~b}$ shows a clear change in the transmitted probe beam by the pump beam action just in the forward direction of the two-wave mixing. It is worth mentioning that an unbalanced distribution in the composition or structure of the film could be responsible for the dispersion and asymmetry in the nonlinear data plotted in Figure $7 \mathrm{~b}$ [55]. From Figure 7b, it can be seen that the maximum transmittance of the probe beam can be obtained for an angle of polarization of $45^{\circ}$, which is in good agreement with the fact that parallel or orthogonal polarizations between the incident beams do not generate any induced birefringence. We observed in our screen a good contrast in an irradiance fringe pattern by using a microscope objective located in the interaction region of the beams in absence of the sample in the experimental setup. The generation of a diffracting grating was expected; however, the absence of a self-diffraction signal was verified in our experiment with nanosecond pulses. The main physical mechanism responsible of the nonlinearity of index seems to be a dynamic thermal effect with instability in a modulation of an induced birefringence and diffraction grating.

It is clear from Figure 1 that different interferometric effects can be induced by the two-wave mixing in the forward or backward configuration associated to the superposition of the pump and probe beams. Then, we experimentally confirmed the observed nonlinear optical behavior in the transmitted Kerr measurements just by switching the position of the bilayer sample in the experiments in a standard two-wave mixing experiment.

The reproducibility of the data plotted in Figures 5-7 was guaranteed for a pulse repetition rate of $1 \mathrm{~Hz}$. Comparative experiments in carbon MWCNT pointed out that the participation of dynamic thermal transport in photothermal effects induced by nanosecond pulses at a $532 \mathrm{~nm}$ wavelength corresponds to a decay time of about $500 \mathrm{~ms}$ [56,57].

For further investigation of the contrast in the nonlinear Kerr transmittance with potential modulation of the nanosecond third-order nonlinear optical behavior of the bilayer sample, we analyzed a standard two-wave mixing interaction by Equation (1). Figure 8 shows numerical results describing the Kerr transmittance in the bidirectional propagation of the beams in the bilayer film interacting in a two-wave mixing.

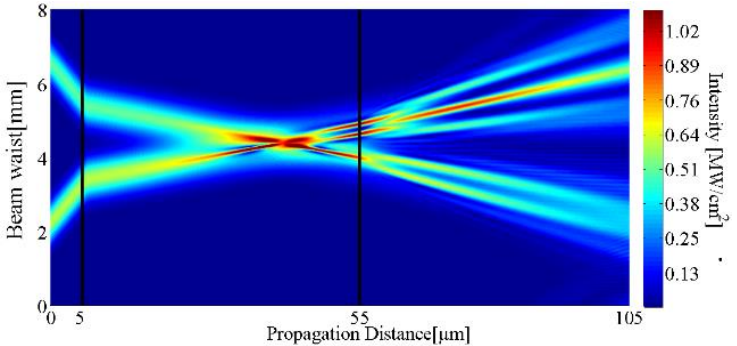

(a)

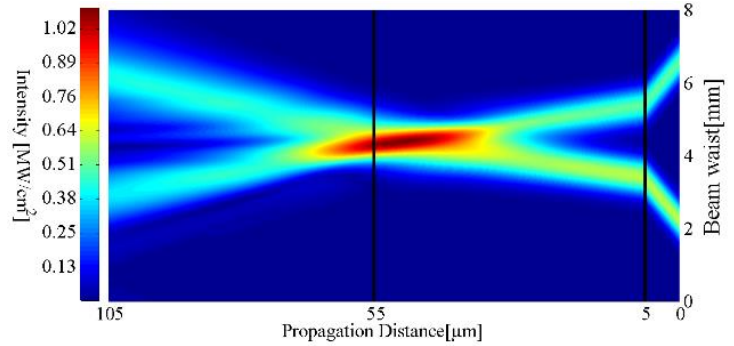

(b)

Figure 8. Numerical results of the propagation of a degenerated two-wave mixing in the studied bilayer sample at $532 \mathrm{~nm}$ wavelength: (a) forward direction; the beams initially interacts in the MWCNT film and then in the Pt-MWCNT; (b) backward direction; the beams initially interacts in the Pt-MWCNT film and then in the MWCNT.

It can be stated that the maximum peak of irradiance in the sample can be obtained for parallel polarizations between the incident beams, because this condition promotes the maximum contrast in the irradiance pattern generated in the sample by constructive interference. From Figure $8 \mathrm{a}, \mathrm{b}$, remarkable differences in the Kerr transmittance obtained by the propagation of the probe beam in the forward and backward direction can be clearly observed in the two-wave mixing interaction. These conditions are associated to the participation of the two-photon absorption in the Pt-MWCNT that results in a depletion of the incident beams before an optical Kerr effect can be excited in the MWCNT. 
A noticeable self-focusing effect occurs in the MWCNT when this layer initially interacts with the two-wave mixing, and it promotes a modulation of the polarization that derives in a Kerr transmittance that can be observed in an optical Kerr gate.

Regarding the results demonstrated in Figures 6-8, nonlinear optical Kerr functions induced by ultrafast optical irradiance or polarization effects can be considered by hierarchical nanostructures. Researchers have previously reported important changes in the mechanical, electronic and optical properties of hybrid nanostructures because of the inclusion of CNT $[58,59]$. In addition, CNT can produce flexural strength in advanced nanocomposites as a function of their concentration of the tubes [60] that could be employed for the instrumentation of optomechanical signals. The density of CNT-based samples receives an important contribution from their thermo-mechanic and photo-physical characteristics [61]. The electromagnetic properties in the hierarchical composites can be correlated to hierarchical structure in good agreement with estimations related to CNT samples [62]. We highlight in this work that the polarization and phase-change in nonlinear optical signals in propagation through CNT can be modulated by orientation-selectable optical effects. Immediate applications of the influence of third-order nonlinear optical properties on hierarchical nanostructures can be considered for developing multifunctional sensors and actuators that are able to control photonic signals. The possibility of low-dimensional interconnections modulated by the vectorial nature of light in quantum systems can be contemplated. An advantage of these findings is the simplicity of the working principle based on the vectorial nature of light and the selectivity of hierarchical nanostructures for controlling nonlinear optical phenomena.

\section{Conclusions}

The impact of platinum decoration in the orientation-selectable nonlinear optical response of hierarchical carbon nanostructures was analyzed. A unidirectional modulation of optical polarization in a bilayer sample was demonstrated by an optical circuit with a bidirectional optical Kerr gate. A strong dependence on the polarization of the induced third-order phenomena in the nanostructures allowed us to systematically control electronic signals in the nanotube networks integrating an electronic circuit. A two-wave mixing method was used to modulate interferometrically controlled laser pulses by third-order optical nonlinearities in the nanostructures. The switching of the optical signals was assisted by the rotation of the angle between the planes of linear polarization of the incident beams in the sample. The experiments were carried out far from the nanosecond ablation threshold of the samples in single-pulse mode. This work highlights the possibility of tailoring polarization-selectable effects in nanosystems by nonlinear optical phenomena. Potential applications for developing signal processing functions driven by nonlinearities induced by light in low-dimensional systems can be considered. The collective behavior exhibited by carbon-based nanostructures with applications for designing nanophotonic platforms driven by nonlinear optical signals can be envisioned.

Author Contributions: Analysis of optical experiments, S.M.-B.; preparation, decoration and morphology characterization of the samples, C.M.-Z.; mechanical studies, J.P.C.-L.; evolution of the nonlinear optical signals during experiments, C.C.-D.; design of hierarchical vectorial functions, C.L.M.-G.; elucidated and evaluated the nonlinear optical properties and designed the paper, C.T.-T. The manuscript was written through contribution of all authors. All authors have read and agreed to the published version of the manuscript.

Funding: The authors kindly acknowledge the financial support from the Instituto Politécnico Nacional, COFAA-IPN, Universidad Politécnica del Valle de México, Universidad Politécnica del Bicentenario, Tecnológico de Estudios Superiores de Coacalco and from the Consejo Nacional de Ciencia y Tecnología (CB-2015-251201).

Acknowledgments: The authors kindly acknowledge to Instituto Politécnico Nacional, Comisión de Operación y Fomento de Actividades Académicas del Instituto Politécnico Nacional, Universidad Politécnica del Valle de México, Universidad Politécnica del Bicentenario, Tecnológico de Estudios Superiores de Coacalco and CONACyT. The authors are also thankful to the Central Microscopy facilities of the CNMN-IPN.

Conflicts of Interest: The authors declare no conflict of interest. 


\section{References}

1. Di Ventra, M.; Evoy, S.; Heflin, J. Carbon Nanotubes. In Introduction to Nanoscale Science and Technology; Kluwer Academic Publishers: Ottawa, ON, Canada, 2004; Volume 1, pp. 137-181.

2. Zhao, Q.; Wood, J.R.; Wagner, H.D. Stress fields around defects and fibers in a polymer using carbon nanotubes as sensors. Appl. Phys. Lett. 2001, 78, 1748-1750. [CrossRef]

3. Ma, L.; Wang, J.; Yip, J.; Ding, F. Mechanism of Transition-Metal Nanoparticle Catalytic Graphene Cutting. J. Phys. Chem. Lett. 2014, 5, 1192-1197. [CrossRef] [PubMed]

4. Kamino, T.; Yaguchi, T.; Konno, M.; Hashimoto, T. In situ high temperature TEM observation of interaction between multi-walled carbon nanotube and in situ deposited gold nano-particles. J. Electron. Microsc. 2005, 54, 461-465. [CrossRef] [PubMed]

5. Gao, Y.; Zhang, X.; Li, Y.; Liu, H.; Wang, Y.; Chang, Q.; Song, Y. Saturable absorption and reverse saturable absorption in platinum nanoparticles. Opt. Commun. 2005, 251, 429-433. [CrossRef]

6. Islam, M.A.; Bhuiya, M.A.K.; Islam, M.S. A review on chemical synthesis process of platinum nanoparticles. Asia Pac. J. Energy Environ. 2014, 1, 107-120. [CrossRef]

7. Sharma, G.; Kumar, D.; Kumar, A.; Al-Muhtaseb, A.H.; Pathania, D.; Naushad, M.; Mola, G.T. Revolution from monometallic to trimetallic nanoparticle composites, various synthesis methods and their applications: A review. Mater. Sci. Eng. C 2017, 71, 1216-1230. [CrossRef]

8. Iolanda, D.B.; Avvisati, G.; Chen, C.; Avila, J.; Asensio, M.C.; Hu, K.; Ito, Y.; Hines, P.; Lipton-Duffin, J.; Rintoul, L.; et al. Topology and doping effects in three-dimensional nanoporous graphene. Carbon 2018, 131, 258-265.

9. Gao, Y.; Shiue, R.-J.; Gan, X.; Li, L.; Peng, C.; Meric, I.; Wang, L.; Szep, A.; Walker Jr., D.; Hone, J.; et al. High-Speed Electro-Optic Modulator Integrated with Graphene-Boron Nitride Heterostructure and Photonic Crystal Nanocavity. Nano Lett. 2015, 15, 2001-2005. [CrossRef]

10. Wagner, P.; Ivanovskaya, V.V.; Rayson, M.J.; Briddon, P.R.; Ewels, C.P. Mechanical properties of nanosheets and nanotubes investigated using a new geometry independent volume definition. J. Phys. Condens. Matter. 2013, 25, 1-5. [CrossRef]

11. Vigueras-Santiago, E.; Hernández-Lopez, S.; Camacho-Lopez, M.A.; Reyes-Contreras, D.; Farías-Mancilla, R.; Flores-Gallardo, S.G.; Hernández-Escobar, C.A.; Zaragoza-Contreras, E.A. Optical properties of carbon nanostructures produced by laser irradiation on chemically modified multi-walled carbon nanotubes. Opt. Laser Technol. 2016, 84, 53-58. [CrossRef]

12. Fazelzadeh, S.A.; Ghavanloo, E. Nonlocal anisotropic elastic shell model for vibrations of single-walled carbon nanotubes with arbitrary chirality. Compos. Struct. 2012, 94, 1016-1022. [CrossRef]

13. Baloch, K.H.; Voskanian, N.; Bronsgeest, M.; Cumings, J. Remote Joule heating by a carbon nanotube. Nat. Nanotechnol. 2012, 7, 316-319. [CrossRef] [PubMed]

14. Sinha, N.; Yeow, J.T.-W. Carbon Nanotubes for Biomedical Applications. IEEE Trans. Nanobiosci. 2005, 4, 180-195. [CrossRef] [PubMed]

15. Torti, S.V.; Byrne, F.; Whelan, O.; Levi, N.; Ucer, B.; Schmid, M.; Torti, F.M.; Akman, S.; Liu, J.; Ajayan, P.M.; et al. Thermal ablation therapeutics based on $\mathrm{CN}(\mathrm{x})$ multi-walled nanotubes. Int. J. Nanomed. 2007, 2, 707-714.

16. Paul, R.; Maity, A.; Mitra, A.; Kumbhakar, P.; Mitra, A. Synthesis and study of optical and electrical characteristics of a hybrid structure of single wall carbon nanotubes and silver nanoparticles. J. Nanopart. Res. 2011, 13, 5749-5757. [CrossRef]

17. BelBruno, J.J. Nanomaterials in sensors. Nanomaterials 2013, 3, 572-573. [CrossRef]

18. García-Merino, J.A.; Jiménez-Marín, E.; Mercado-Zúñiga, C.; Trejo-Valdez, M.; Vargas-García, J.R.; Torres-Torres, C. Quantum and bistable magneto-conductive signatures in multiwall carbon nanotubes decorated with bimetallic Ni and Pt nanoparticles driven by phonons. OSA Contin. 2019, 2, 1285-1295. [CrossRef]

19. Hernández-Acosta, M.A.; Martínez-Gutiérrez, H.; Martínez-González, C.L.; Torres-SanMiguel, C.R.; Trejo-Valdez, M.; Torres-Torres, C. Fractional and chaotic electrical signatures exhibited by random carbon nanotube networks. Phys. Scr. 2018, 93, 125801. [CrossRef]

20. Yoshida, K.; Arai, S.; Sasaki, Y.; Tanaka, N. Catalytic oxidation of carbon nanotubes with noble metal nanoparticles. Micron 2015, 76, 19-22. [CrossRef] 
21. Ispasoiu, R.G.; Balogh, L.; Varnavski, O.P.; Tomalia, D.A.; Goodson, T., III. Large optical limiting from novel metal-Dendrimer nanocomposite materials. J. Am. Chem. Soc. 2000, 122, 11005-11006. [CrossRef]

22. Qu, S.; Song, Y.; Liu, H.; Wang, Y.; Gao, Y.; Liu, S.; Zhang, X.; Li, Y.; Zhu, D. A theoretical and experimental study on optical limiting in platinum nanoparticles. Opt. Commun. 2002, 203, 283-288. [CrossRef]

23. Henglein, A.; Ershov, B.G.; Malow, M. Absorption spectrum and some chemical reactions of colloidal platinum in aqueous solution. J. Phys. Chem. 1995, 99, 14129-14136. [CrossRef]

24. Philip, R.; Kumar, G.R.; Sandhyarani, N.; Pradeep, T. Picosecond optical nonlinearity in monolayer-protected gold, silver, and gold-silver alloy nanoclusters. Phys. Rev. B Cov. Cond. Matt. Mat. Phys. 2000, 62, 13160-13166. [CrossRef]

25. Maiti, A. Application of carbon nanotubes as electromechanical sensors-Results from first-principles simulations. Phys. Status Solidi B 2001, 226, 87-93. [CrossRef]

26. Mercado-Zúñiga, C.; Torres-Torres, C.M.; Torres-Mancera, T.; Vargas-García, J.R.; Torres-Martínez, R. Dynamically configurable nonlinear optical switching based on vertically aligned carbon nanotubes. Mater. Res. 2016, 19, 52-58.

27. Wildgoose, G.G.; Banks, C.E.; Compton, R.G. Nanoparticles and Related Materials Supported on Carbon Nanotubes: Methods and Applications. Nano Micro Small J. 2006, 2, 182. [CrossRef] [PubMed]

28. You, J.W.; Bongou, S.R.; Bao, Q.; Panoiu, N.C. Nonlinear optical properties and applications of 2D materials theoretical and experimental aspects. Nanophotonics 2019, 8, 63-97. [CrossRef]

29. Singh, E.; Srivastava, R.; Kumar, U.; Katheria, A.D. Carbon Nanotube A Review on Introduction, Fabrication Techniques and Optical Applications. AIP Conf. Proc. 2017, 4, 120-126.

30. Yamashita, S. Nonlinear optics in carbon nanotube, graphene, and related 2D materials. APL Photonics 2019, 4, 034301. [CrossRef]

31. Elim, H.I.; Zhu, Y.; Sow, C.H. Length Dependence of Ultrafast Optical Nonlinearities in Vertically Aligned Multiwalled Carbon Nanotube Films. J. Phys. Chem. 2016, 120, 17733-17738. [CrossRef]

32. Ko, W.Y.; Su, J.W.; Guo, C.H.; Lin, K.J. Extraordinary mechanical flexibility in composite thin films composed of bimetallic AgPt nanoparticle-decorated multi-walled carbon nanotubes. Carbon 2012, 50, $2244-2251$. [CrossRef]

33. Park, S.J.; Ok, J.G.; Park, H.J.; Lee, K.T.; Lee, J.H.; Kim, J.D.; Chog, E.; Baac, H.W.; Kang, S.; Guo, L.J.; et al. Modulation of the effective density and refractive index of carbon nanotube forests via nanoimprint lithography. Carbon 2017, 129, 8-14. [CrossRef]

34. Jiménez-Marín, E.; Torres-Torres, C.; Mercado-Zúñiga, C.; Vargas-García, J.R.; Trejo-Valdez, M.; Cervantes-Sodi, F.; Torres-Martínez, R. Interferometrically-controlled electrical currents in carbon nanotubes coated by platinum nanoparticles. Opt. Laser Technol. 2016, 85, 35-40. [CrossRef]

35. Mercado-Zúñiga, C.; Torres-Torres, C.; Trejo-Valdez, M.; Torres-Martínez, R.; Tarrago-Velez, S.; Cervantes-Sodi, F.; Vargas-García, J.R. Mechanooptic regulation of photoconduction in functionalized carbon nanotubes decorated with platinum. Int. J. Photoenergy 2014, 542658. [CrossRef]

36. Piña-Díaz, A.J.; Torres-Torres, D.; Trejo-Valdez, M.; Torres-SanMiguel, C.R.; Martínez-González, C.L.; Torres-Torres, C. Decision making two-wave mixing with rotating TiO2-supported Au-Pt nanoparticles. Opt. Laser Technol. 2019, 119, 105638. [CrossRef]

37. Castro-Chacón, J.H.; Khomenko, A.V.; Rangel-Rojo, R. Phase matched vectorial three-wave mixing in isotropic Kerr media. Opt. Commun. 2009, 282, 1422-1426. [CrossRef]

38. Boyd, R.W. Nonlinear Optics; Academic Press: San Diego, CA, USA, 2009.

39. Bornacelli, J.; Torres-Torres, C.; Silva-Pereyra, H.G.; Rodríguez-Fernández, L.; Avalos-Borja, M.; Cheang-Wong, J.C.; Oliver, A. Nanoscale influence on photoluminescence and third order nonlinear susceptibility exhibited by ion-implanted Pt nanoparticles in Silica. Methods Appl. Fluoresc. 2017, 5, 025001. [CrossRef]

40. Dresselhaus, M.S.; Dresselhaus, G.; Saito, R. Physics of carbon nanotubes. Carbon 1995, 33, 883-891. [CrossRef]

41. Qian, D.; Wagner, G.J.; Liu, W.K.; Yu, M.F.; Ruoff, R.S. Mechanics of carbon nanotubes. Appl. Mech. Rev. 2002, 55, 495-533. [CrossRef]

42. Li, C.; Chou, T.W. A structural mechanics approach for the analysis of carbon nanotubes. Int. J. Solids Struct. 2003, 40, 2487-2499. [CrossRef]

43. Fan, C.W.; Liu, Y.Y.; Hwu, C. Finite element simulation for estimating the mechanical properties of multi-walled carbon nanotubes. Appl. Phys. A 2009, 95, 819-831. [CrossRef] 
44. Brown-Theodore, L.; Le May, H.E., Jr.; Bursten-Bruce, E. Chemestry: The Central Science, 11st ed.; Pearson Prentice Hall: Upper Saddle River, NJ, USA, 1997; Volume 8, pp. 412-413.

45. Yakobson, B.I.; Brabec, C.J.; Bernholc, J. Nanomechanics of carbon nanotubes: Instabilities beyond linear response. Phys. Rev. Lett. 1996, 76, 2511-2514. [CrossRef] [PubMed]

46. Odegard, G.M.; Gates, T.S.; Nicholson, L.M.; Wise, K.E. Equivalent-Continuum Modeling with Application to Carbon Nanotubes; National Aeronautics and Space Administration, Langley Research Center: Hampton, VA, USA, 2002; pp. 1-13.

47. Robertson, D.H.; Brenner, D.W.; Mintmire, J.W. Energetics of nanoscale graphitic tubules. Phys. Rev. Lett. B 1992, 45, 12592-12595. [CrossRef]

48. Popov, E.P. Stability of Equilibrium: Columns. In Mechanics of Materials; Loret, B., Subhash, G., Eds.; Prentice Hall Inc.: Upper Saddle River, NJ, USA, 1990; pp. 574-578.

49. He, X.Q.; Eisenberger, M.; Liew, K.M. The effect of van der Waals interaction modelling on the vibration characteristics of multi-walled carbon nanotubes. J. Appl. Phys. 2006, 100, 124317. [CrossRef]

50. Strozzi, M.; Pellicano, F. Linear vibrations of triple-walled carbon nanotubes. Math. Mech. Solids 2018, 23, 1456-1481. [CrossRef]

51. Wong, E.W.; Sheehan, P.E.; Lieber, C.M. Nanobeam mechanics: Elasticity, strength, and toughness of nanorods and nanotubes. Sciences 1997, 277, 1971-1975. [CrossRef]

52. Popov, V.N.; Van Doren, V.E.; Balkanski, M. Elastic properties of single-walled carbon nanotubes. Phys. Rev. B 2000, 61, 3078-3084. [CrossRef]

53. Kudin, K.N.; Scuseria, G.E.; Yakobson, B.I. C2F, BN and C nanoshell elasticity from ab initio computations. Phys. Rev. B 2001, 64, 235406. [CrossRef]

54. Shimamoto, D.; Sakurai, T.; Itoh, M.; Kim, Y.; Hayashi, T.; Endo, M. Nonlinear optical absorption and reflection of single wall carbon nanotube thin films by Z-scan technique. Appl. Phys. Lett. 2008, 92, 1-14. [CrossRef]

55. Feria-Reyes, E.; Torres-Torres, C.; Martínez-Gutiérrez, H.; Morales-Bonilla, S.; Torres-Martínez, R.; Trejo-Valdez, M.; Urriolagoitia-Calderón, G. Mechano-optical modulation and optical limiting by multiwall carbon nanotubes. J. Mod. Opt. 2013, 60, 1321-1326. [CrossRef]

56. Hernández-Acosta, M.A.; Martines-Arano, H.; Soto-Ruvalcaba, L.; Martínez-González, C.L.; Martínez-Gutiérrez, H.; Torres-Torres, C. Fractional thermal transport and twisted light induced by an optical two-wave mixing in single-wall carbon nanotubes. Int. J. Thermal Sci. 2020, 147, 106136. [CrossRef]

57. García-Merino, J.A.; Martínez-González, C.L.; Torres-SanMiguel, C.R.; Trejo-Valdez, M.; Martínez-Gutiérrez,H.; Torres-Torres, C. Photothermal, photoconductive and nonlinear optical effects induced by nanosecond pulse irradiation in multi-wall carbon nanotubes. Mat. Sci. Eng. B 2015, 194, 27-33. [CrossRef]

58. Anand, B.; Kaniyoor, A.; Sai, S.S.S.; Philip, R.; Ramaprabhu, S. Enhanced optical limiting in functionalized hydrogen exfoliated graphene and its metal hybrids. J. Matt. Chem. C 2013, 15, 2773-2780. [CrossRef]

59. Ruoff, R.S.; Qian, D.; Liu, W.K. Mechanical properties of carbon nanotubes: Theoretical predictions and experimental measurements. C. R. Phys. 2003, 4, 993-1008. [CrossRef]

60. Roche, S. Carbon Nanotubes: Exceptional mechanical and electronic properties. Ann. Chim. Sci. Mater. 2000, 25, 529-532. [CrossRef]

61. Geng, Y.; Liu, M.Y.; Li, J.; Shi, X.; Kim, J. Effects of surfactant treatment on mechanical and electrical properties of CNT/epoxy nanocomposites. Compos. Part A Appl. Sci. Manuf. 2008, 39, 1876-1883. [CrossRef]

62. Ku-Herrera, J.J.; La Saponara, V.; Avilés, F. Selective damage sensing in multiscale hierarchical composites by tailoring the location of carbon nanotubes. J. Intell. Mater. Syst. Struct. 2018, 29, 553-562. [CrossRef]

(C) 2020 by the authors. Licensee MDPI, Basel, Switzerland. This article is an open access article distributed under the terms and conditions of the Creative Commons Attribution (CC BY) license (http://creativecommons.org/licenses/by/4.0/). 